\title{
Attention bands in absolute identification
}

\author{
R. DUNCAN LUCE, DAVID M. GREEN, and DANIEL L. WEBER \\ Harvard University, Cambridge, Massachusetts 02138
}

\begin{abstract}
If both the number of one-dimensional signals and their range are sufficiently large (about 7 and $20 \mathrm{~dB}$ for loudness), the information transmitted in absolute identification is not much increased by increasing either variable (Miller, 1956; Braida \& Durlach, 1972). The data can be represented in terms of Thurstonian discriminal dispersions in which the variance is proportional to the square of the signal range in decibels (Durlach \& Braida, 1969; Gravetter \& Lockhead, 1973), but it is by no means obvious what sorts of mechanisms would lead to this model. An alternative is proposed, namely, that there is a roving attention band, about 10 to $15 \mathrm{~dB}$ wide, such that signals falling within the band are represented by a sensory sample size about an order of magnitude larger than when the same signal falls outside the band. With reasonable choices for parameters, including the subjective continuum growing as a power function of intensity with an exponent about .3, this nicely accounts for the data. In an attempt to examine the change of performance with range, we replicated the Braida-Durlach experiment with many additional points. These data are not, however, adequate to decide between the two models.
\end{abstract}

When 10 or more one-dimensional signals are spread over a sufficiently large range (e.g., $20 \mathrm{~dB}$ or more in auditory intensity), subjects in absolute identification (AI) experiments do not perform as well as one would anticipate from data on comparable pairs of signals. No satisfactory explanation has been given. The purpose of this paper is to propose one.

The earliest systematic evidence demonstrating this are Garner's (1953) and Pollack's (1952) auditory data, which, together with similar data from other modalities, were widely disseminated via Miller's (1956) classic "The magical number seven, plus or minus two: Some limits on capacity for processing information." Garner used from 4 to 20 pure tones of intensities spaced equally in decibels over a 95-dB range. To a first approximation, performance, as measured by the information transmitted, grows linearly to about 2.3 bits, after which it remains constant. The data points are shown in Figure 1.

This is not what one would expect if a Thurstonian model were correct. If each signal is represented by a random variable that is independent of the ensemble of signals used and if responses are determined by categories whose boundaries lie somewhere between the mean representations of successive signals, then the information transmitted should grow much more steadily.

A recent study by Braida and Durlach (1972) presents another equally inexplicable, but we suspect related, finding. In the part of their study of interest here, they used 10 pure tone signals spaced equally in decibels over their range, which was varied from

This work was supported in part by a National Science Foundation grant to the University of California, Irvine, and one to Harvard University.

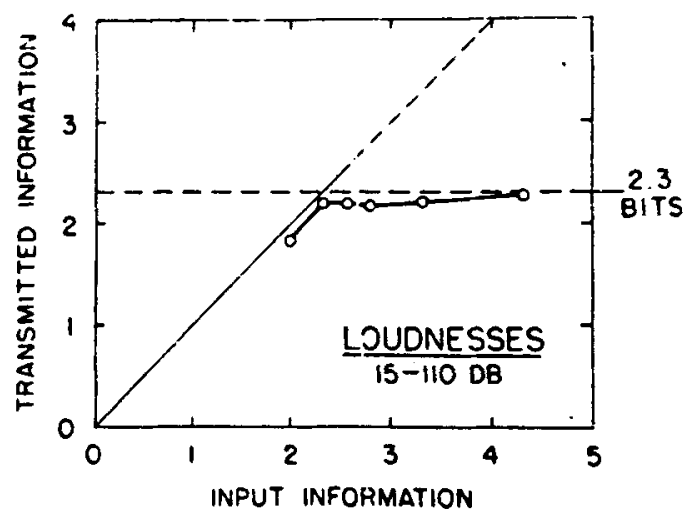

Figure 1. Information transmitted vs. information input in loudness absolute identification for a 95-dB range. The input was controlled through the number of equally probable signals. The data are from Garner (1953); the figure is from Miller (1956, Figure 2).

2.25 to $54 \mathrm{~dB}$. Their analysis, described in detail in the Appendix, involved computing a $\mathrm{d}^{\prime}$ measure for successive pairs of signals and summing it over all 10 signals to give an overall measure, $\Delta^{\prime}$, of accuracy. For small ranges it grows linearly, but gradually it decelerates-see the data points of Figure 2.

Durlach and Braida (1969) had postulated a Thurstonian model in which the mean scale value grows linearly in decibels. This implies that $\Delta^{\prime}$ should grow linearly with range in decibels, which is wrong. We have verified that a power function growth does not predict the data either. To deal with this, they suggested, as did Gravetter and Lockhead (1973) independently, that the variance of the signal representation grows linearly with the square of the range in decibels. Although partially accounting for their results-it fails to explain why comparable magnitude estimation (ME) results lie systematically 


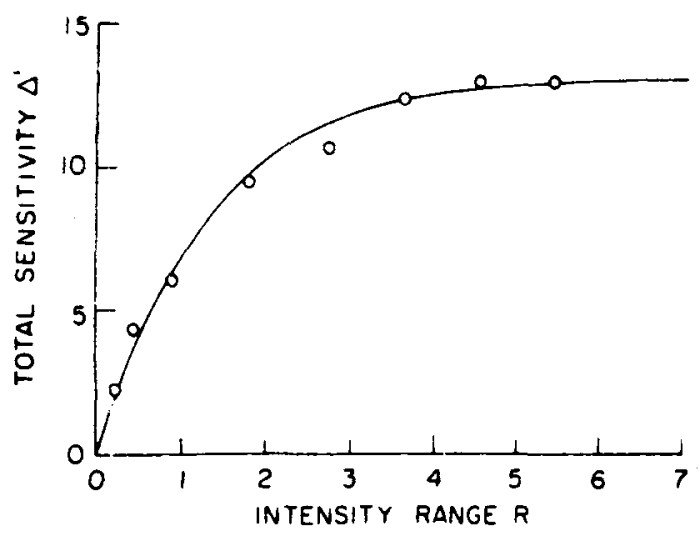

Figure 2. Total sensitivity vs. intensity range $R(=d B / 10)$ for loudness absolute identification as reported by Braida and Durlach (1972, Figure 4d).

below the AI ones (see Braida \& Durlach, 1972, Figure $6 f$ ) or why $d^{\prime}$ is appreciably larger at the extremes of the range than in the middle (see their Figure 2)-we do not consider it a very satisfying explanation. No reason is offered either as to why the range should directly affect the variability of the representation of a signal or as to why it should be the square of the range in decibels. The account seems wholly ad hoc.

\section{AN ATTENTION MODEL}

Luce and Green (1972, 1974) have discussed psychophysical models in which the signal is represented on each of $\mathbf{J}$ channels as independent Poisson processes with a parameter $\mu$ that is an increasing function of signal intensity. They have argued that if signals are of sufficient duration, subjects probably take a fixed sample size $\boldsymbol{x}$ on each of $\mathrm{J}$ channels and use it to estimate the Poisson parameter, and hence the intensity, by observing the total time required to get the sample. This timing behavior is to be contrasted with counting the number of firings during a fixed time interval, which appears to occur when the signals are very brief. Later work (Green \& Luce, 1974) on ME led us to propose that the size of the total sample, $\mathrm{k}=\mathrm{Jx}$, may very well not be a constant when the signals span a sufficiently large range. The intuition is that the CNS cannot fully monitor all 30,000 peripheral auditory neurons and that it distributes its attention by fully monitoring a band between 10 and $20 \mathrm{~dB}$ at a given frequency and by monitoring the rest of the range much less completely. Roughly, we estimated the two sample sizes to differ by a factor of from 5 to 10. We also were led to suggest that the attention interval tends to be centered at the location of the preceding signal, although, by altering the distribution of signals from a uniform one, this was shown not to be inevitable.
The last study, along with several others (Luce \& Green, 1972, 1974b), also suggests that the growth of the Poisson parameter with sound intensity is approximately a power function with the exponent $y$ in the range from .15 to .60 over subjects, usually between .20 and .30 . We assume this form.

The following AI model was programmed for computation.'

(1) The $\mathrm{n}$ signals are spaced equally in decibels over a range of $\mathbf{R} \mathrm{dB}$.

(2) Each signal is equally likely to be presented.

(3) The attention interval is $\mathrm{A} \mathrm{dB}$ wide independent of its location.

(4) If a signal lies outside the attention inverval, the sample size is $K_{0}$; if it lies within the interval, the sample size is $K_{1}\left(K_{1}>K_{0}\right)$.

(5) The Poisson parameter is a power function, with exponent $\gamma$, of the ratio of signal intensity to threshold intensity.

(6) Category boundaries are located at the geometric mean of the Poisson parameters of successive signals.

(7) The subject responds by giving the category number containing the estimate of the Poisson parameter computed from the sample.

Let $P_{m}\left(j \mid I_{j}\right)$ denote the probability that the response to the signal of intensity $I_{i}, i=1, \cdots, n$, is category $\mathrm{j}$ or less when the sample size is $K_{\mathrm{m}}, \mathrm{m}=$ 0,1 . Using the normal approximation to the gamma distribution and defining

$$
\theta(i, j)=1-10^{\frac{10 \gamma R}{n-1}\left(i-j-\frac{1}{2}\right)}
$$

it is easy to see that

$$
P_{m}\left(j \mid I_{i}\right) \cong \int_{-\infty}^{K_{m} \theta(i, j)} \Phi(0,1), \quad m=0,1
$$

where $\Phi(0,1)$ denotes the normal density with mean 0 and variance 1 . Since a randomly presented signal falls in the attention interval with probability $A / R$, we see that average response probability is

$$
\bar{P}\left(j \mid I_{i}\right)= \begin{cases}P_{1}\left(j \mid I_{i}\right) \frac{A}{R}+P_{0}\left(j \mid I_{i}\right)\left(1-\frac{A}{R}\right), & A<R \\ P_{1}\left(j \mid I_{i}\right), & A \geqslant R .\end{cases}
$$

And the information transmitted is given by

$$
\left.T=-\frac{1}{n} \Sigma_{i} \Sigma_{j} \overline{P(j} \mid I_{i}\right) \log _{2} \frac{\bar{P}\left(j \mid I_{i}\right)}{\bar{P}(j)} .
$$

If we convert these average probabilities into $z$ scores and substitute into Equation 5 of the Appendix, we compute Braida and Durlach's $\Delta^{\prime}$. 


\section{FIT OF THE MODEL TO DATA}

The parameters $\mathbf{n}$ and $\mathbf{R}$ are determined by the experiment. This leaves the parameters $A, K_{0}, K_{1}$, and $\gamma$ to be estimated; however, we were limited in our choices by earlier estimates of all of them. On the basis of ME data for loudness, we felt that A should lie between 10 and $20 \mathrm{~dB} ; \mathrm{K}_{0}$ should be in the range 10 to 30 , and $K_{1}$ in the range 60 to 200 ; and on the basis of a number of different auditory intensity experiments, we felt that the average value of $r$ should lie between .2 and .3. With these constraints in mind, we attempted to fit both the Garner and Braida-Durlach data. The former was easily fit well, as can be seen in Figure 3. The latter proved to be much more tricky, and the estimates are much less sure. The problem is illustrated by the two sets of parameter values shown in Figure 4.

The growth of the theoretical $\Delta^{\prime}$ with $R$ is nonmonotonic: for ranges up to the attention band, the growth is linear, after which there is a rather sharp drop followed by a more gradual rise. The nature of the fall and rise depends primarily on $\mathbf{K}_{1} / \mathbf{K}_{0}$ and $\gamma$. The data points are simply not sufficiently closely spaced in the region of our estimate of $\mathbf{A}$ (and are averaged over three subjects) to be able to test the predicted dip of the theory.

We mention two further aspects of the BraidaDurlach data in relation to this model. First, they found for each range that the values of $d^{\prime}$ near both the least and the most intense signals were larger than in the midrange. They refer to this as an "edge effect." We would expect this if the attention interval were not distributed uniformly over the range, but rather tended more often to be located at the maximum and minimum of the range. Why this should happen is not, however, clear.

Second, the curve of $\Delta^{\prime}$ computed from ME lies appreciably below that from AI. In discussing

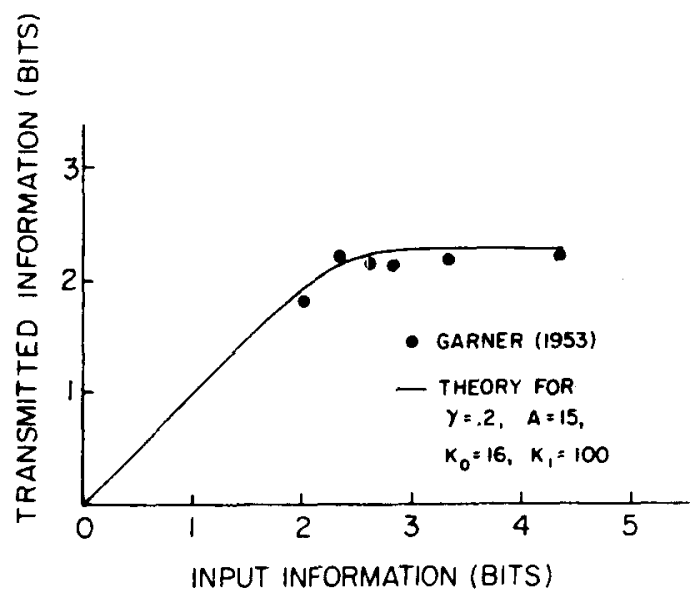

Figure 3. Garner's data as fit by the model described in the text with the four parameter values shown.
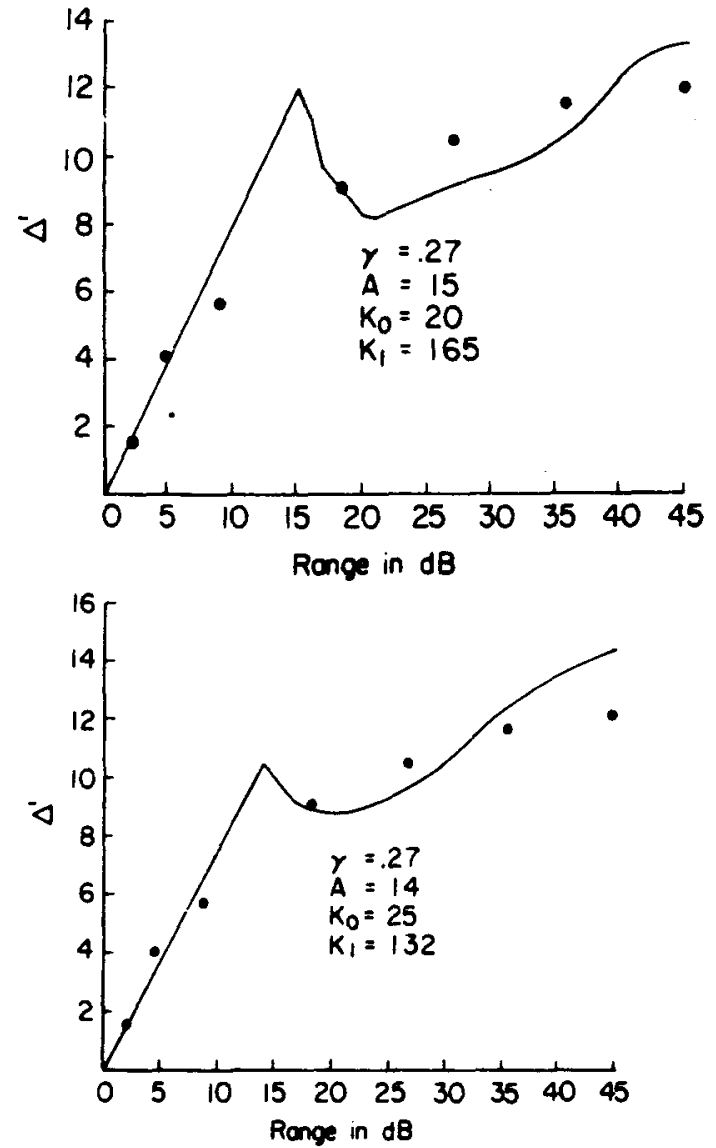

Figure 4. Braida and Durlach's data as fit by the model with two sets of four parameter values.

sequential and drift effects in magnitude estimation, Luce and Green (1974a) were led to postulate that the sample size in $\mathrm{ME}$ is half of that in an $\mathrm{AI}$ experiment because in order to carry out the computations of ratios on successive trials the subject computes two independent estimates of each signal. This means that the standard deviation of the estimate is $1 / \sqrt{2}$ larger than in $\mathrm{AI}$, which accounts for the observed difference. Usually, feedback is used in $\mathrm{AI}$ and not in ME, and so it is often thought to account for the differences in observed variability; however, Braida and Durlach did not employ feedback in either the AI or the ME experiments used for this comparison.

\section{ABSOLUTE IDENTIFICATION AS A FUNCTION OF SIGNAL RANGE}

Since the attention band model suggests some rather perculiar behavior for ranges between 10 and $20 \mathrm{~dB}$ and since the Braida-Durlach data are not very detailed in that region and are averaged over subjects, we decided to replicate their experiment with more values of the range, especially in that region. 


\section{Procedure}

Four observers, all Harvard students, were run individually or in groups of two or three in a quiet room. They were paid $\$ 2.25 / \mathrm{h}$.

Each intensity range was centered at about $60 \mathrm{~dB}$ SPL, and the 10 signals were equally spaced in decibels. The signals, which were delivered binaurally through TDH-39 earphones, were $1,000-\mathrm{Hz}, 500-\mathrm{msec}$ tones. A digital attenuator controlled the intensity values. The minimum step on the attenuators was $0.25 \mathrm{~dB}$. For all stimulus ranges, the center of the range was as near $60 \mathrm{~dB}$ as could be achieved, $60 \pm 0.125 \mathrm{~dB}$.

Usually, the same range was used throughout the 1,200 trials of a 2-h session, and it was never changed in less than 600 trials. A brief rest was provided after every 100 trials and a longer one after every 300 . Prior to each block of 100 trials, each signal was identified by the numbers 1 through 10 as they were each presented twice in ascending order. For each range, each signal occurred about 120 times.

The observers responded by using a single digit on a 16-key calculator keyboard; they could alter their responses which were displayed visually, prior to registering them. The same electronic display also served to indicate the signal interval and to provide information feedback. All experimental operations were controlled by a PDP-12 computer, which paced the experiment to the group response.

Observer D.W. had 9 weeks and the other three observers had 2 weeks of training in an absolute identification task involving six intensity levels; and then they had 3 days of practice on the 10-signal, 4.5-dB range.

\section{Results}

In working with these data in relation to the two models, we concluded that it is unwise to use the $\Delta^{\prime}$ measure because it is too unstably influenced by estimates of small probabilities. So we have confined our analysis to the amount of information transmitted, which for the attention band model exhibits the same qualitative characteristics in somewhat attenuated form.

The Braida-Durlach model predicts

$$
T=R\left(B R^{2}+C\right)^{-1 / 2},
$$

where $R$ is the range in bels. We selected $B$ and $C$ so as to minimize

$$
\operatorname{RMS}=\left\{\frac{1}{m_{i=1}} \sum_{0, i}^{m}\left(T_{p, i}\right)^{2}\right\}^{1 / 2}
$$

where $T_{0, i}$ and $T_{p, i}$ are the observed and predicted values of the information transmitted for the $\mathrm{ith}$ range.

The equations for the attention band model were given above. As there are four parameters, $\gamma, \mathbf{A}, \mathrm{K}_{\mathbf{0}}$, and $K_{1}$, we used a slightly less than optimal procedure. For each of several plausible values of $\mathbf{A}$, we searched for triples of $\gamma, K_{0}$, and $K_{1}$ that yielded a minimum RMS value; however, we only searched in regions suggested by other estimates of such parameters (these were mentioned above). We found that the data pretty well determined the ratio $K_{1} / K_{0}$, but with that held fixed various $K_{1}, \gamma$ pairs were about equally satisfactory. Thus, the choice of parameters actually
Table 1

Parameters of the Attention Band and Braida-Durlach Models and RMS Measures of Goodness of Fit to AbsoluteIdentification Range Data

\begin{tabular}{lcrrrrrrr}
\hline Sub- & \multicolumn{4}{c}{ Attention Band } & \multicolumn{4}{c}{ Braida-Durlach } \\
ject & $\lambda$ & \multicolumn{1}{c}{$\mathrm{A}$} & $\mathrm{K}_{\mathbf{0}}$ & $\mathrm{K}_{\mathbf{1}}$ & RMS & \multicolumn{1}{c}{ B } & C & RMS \\
\hline D.M. & .271 & 15.8 & 26 & 78 & .087 & .193 & 92.8 & .101 \\
J.C. & .275 & 16.8 & 18 & 72 & .075 & .259 & 82.4 & .076 \\
D.W. & .258 & 10.1 & 11 & 130 & .096 & .500 & 73.4 & .144 \\
J.R. & .330 & $\mathbf{8 . 3}$ & 11 & 116 & .120 & .478 & 51.5 & .093 \\
\hline
\end{tabular}

given is somewhat arbitrary. Finally, we selected that value of $A$ which gave a minimum $R M S$ value, where we only looked at $A$ values which were plausible from a plot of the data.

The resulting estimates and RMS values are shown in Table 1, and the data and theoretical curves are shown in Figure 5. In terms of RMS values, the attention model has a slight edge, especially since the estimates are not fully optimal; however, it has two more parameters. It is exceedingly difficult to tell whether the data have a cusp as predicted.

Although the two models appear about equally satisfactory in accounting for the anomolies in absolute identification data when the range is large, we would argue that the attention band model has several points in its favor and certainly bears more investigation. First, it provides a way of accounting for the edge effect in absolute identification data (a tendency to locate the band at the extreme values) and for the responses in magnitude estimation to be less variable when a signal is close to the preceding one (a tendency to locate the band at the last signal). Second, it is conceptually similar to the critical band concept for frequency, and we must entertain the hypothesis that they are two aspects of the same phenomenon. The tuning curves of the auditory system, for example, suggest that only some small subset of fibers is maximally sensitive to changes in

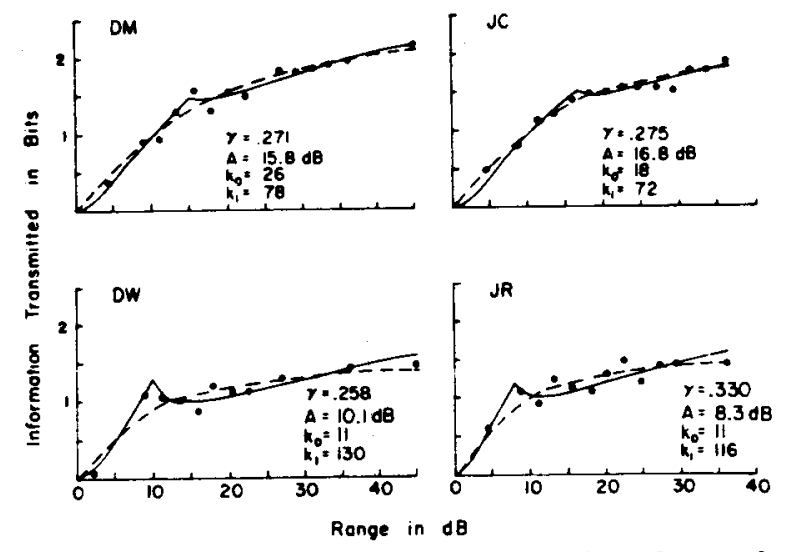

Figure 5. Information transmitted as function of range for four observers together with theoretical predictions of the attention band model (solid line) and the Braida-Durlach model (dashed line). The parameter values shown are for the attention band model. 
frequency from a given frequency and intensity. It therefore makes sense for the central nervous system to monitor intensively this group of fibers. Similarly, a particular subset of fibers is maximally sensitive to changes in intensity at a given frequency and intensity. And so these subsets may also receive differential treatment in intensity discrimination. And third, it seems to us intuitively more satisfactory to have range play an indirect role in the variability of stimuli-increasing the probability that a signal is represented by a small sample - than to postulate a direct effect of range, as in the Braida-Durlach model.

A referee pointed out that in not running a very large range-say $90 \mathrm{~dB}$-we missed an opportunity to test a major difference between the models. Ours predicts that the value of information transmitted grows slowly with large $R$, whereas the BraidaDurlach model is nearly asymptotic by $50 \mathrm{~dB}$. There is, nonetheless, indirect evidence favoring our model. For our four subjects, the average maximum information transmitted is about 1.7 bits (Figure 5), whereas the Garner data for $R=95 \mathrm{~dB}$ and $n=10$ is about 2.2 bits (Figure 1). The difference may be substantially larger than this for the following reason. The parameter values used to fit his data are $\gamma=.2$, $K_{0}=16, K_{1}=100$, which produces a curve substantially below those of subjects D.M. and J.C., which are the two with comparable values of $A$, and so we suspect that had we the relevant data on his subjects they would be substantially below 2 bits at the 50-dB range. This suggests that there is continued growth with range, but clearly the data should be collected to show this.

While the preceding summarizes our arguments as to the virtues of the attention band hypothesis, we must report another analysis of the data that reveals a difficulty in applying it to absolute identification data. The analysis was motivated by a finding in magnitude estimation data, that the coefficient of variation is smaller by a factor of three when the intensity of the preceding stimuli is near the intensity of the present stimulus rather than far. This suggests that we measure in these experiments the percentage of correct judgments conditional on the decibel difference between the present and preceding stimuli. Presumably, if the attention band is located near the last stimulus and the present stimulus is not very different from it, a large sample size results and a higher percentage of correct categorizations should result than if the present stimulus is far from the preceding one. The results did not exhibit this pattern, except, perhaps, for one of the four subjects. Even at the largest ranges employed, the percentage of correct responses was often about the same when successive stimuli were within $10 \mathrm{~dB}$ as when they were more than $20 \mathrm{~dB}$ apart. The major effect is that the extreme stimuli, especially the louder ones, had a higher percentage of correct responses. This means either that the attention hypothesis is wrong or that factors other than the value of the previous stimulus control its location.

\section{APPENDIX \\ THE BRAIDA-DURLACH METHOD OF ANALYSIS}

Let $I_{1}, \cdots, I_{n}$ denote, in ascending order, the $n$ signal intensities of a pure tone; $I_{n}$ was always fixed at $86 \mathrm{~dB}$ SPL. Braida and Durlach's (1972) primary theoretical quantity, called the cumulative sensitivity function $\mathrm{d}^{\prime}\left(\mathrm{I}_{\mathrm{i}} \mathrm{I}_{1}\right)$, is the distance between the means of the RVs associated with $I_{1}$ and $I_{i}$ normalized by their common standard deviation. Because $d^{\prime}$ is obviously additive in their model, they never actually estimate $d^{\prime}\left(I_{i} I_{1}\right)$ but rather a quantity, which for clarity we may denote as

$$
d^{*}\left(I_{i}, I_{1}\right)=\sum_{j=1}^{i-1} d^{\prime}\left(I_{j+1}, I_{j}\right) .
$$

Throughout their paper, the discussion is in terms of the primed quantities; however, all estimates are actually of starred quantities. This distinction does not matter if their model is correct, but does otherwise.

They estimated $d^{*}$ by first averaging the $z$ scores over all relevant response categories for adjacent stimulus intensities and then adding these estimates:

$$
\hat{d}^{*}\left(I_{i}, I_{1}\right)=\sum_{k=1}^{i-1} \frac{1}{n-1} \sum_{j=1}^{n-1}\left[z_{k}\left(p_{j}\right)-z_{k+1}\left(p_{j}\right)\right],
$$

where $p_{j}$ is interpreted as the category boundary separating the $j^{\text {th }}$ from the $(j+1)^{\text {st }}$ response, and because of the equal variance normal postulate, $z_{k}\left(p_{j}\right)$ is defined $b y$,

$$
P\left(j \mid I_{k}\right)=\int_{-\infty}^{z_{k}\left(P_{j}\right)} \Phi(0,1)
$$

To be completely precise, they omitted from Equation 6 all terms involving values of $z$ for which $|z|>2.33$, which corresponds to $P$ values $<.01$ and $>.99$. This surely introduces a bias in the results; judging by some calculations reported in the Appendix of their paper, it is not very serious.

Two other quantities, also of interest, are:

$$
\Delta^{\prime}=d^{\prime}\left(I_{n}, I_{1}\right) \text { and } \Delta^{*}=d^{*}\left(I_{n}, I_{1}\right) .
$$

Some of their empirical results can be summarized as follows:

(1) Plots of $d^{*}\left(I_{i}, I_{1}\right)$ vs. $\log I_{i}$ are approximately linear, but with some deviations which they attribute to deviations from logarithmic assumption and to an edge effect [Figures 1d, 3a, 3b, 4a, 4b of Braida and Durlach (1972)].

(2) The above plot is relatively insensitive to the number n of signals used and their actual distribution so long as the range is fixed (Figures 2 and 3 ).

(3) The plot of $\Delta^{*}$ vs. range in decibels is an increasing function that appears to be approaching an asymptote (Figures 4d and 6f). 
(4) Magnitude estimation data, which are analyzed in an analogous way, yield qualitatively similar results; however, the function relating $\Delta^{*}$ to range is systematically below that for absolute identification (Figure 6f).

It should be noted that by estimating $\mathrm{d}^{\prime}$ by the necessarily additive $d^{*}$, they assume, but do not test, that $d^{\prime}$ is additive. In an appendix, they explore how sensitive their empirical function is to the equal-variance, normaiity assumption of their Thurstonian model, and they find that it is relatively insensitive. Thus, the data are, in fact, consistent with a number of alternative models.

\section{REFERENCES}

Braida. L. D.. \& Durlach, N. I. Intensity perception. II. Resolution in one-interval paradigms. Journal of the Acoustical Society of America, 1972, 51, 483-502.

Durlach, N. I., \& BraidA, L. D. Intensity perception. I. Preliminary theory of intensity resolution. Journal of the Acoustical Society of A merica, 1969, 46, 372-383.

GARNER, W. R. An informational analysis of absolute judgments of loudness. Journal of Experimental Psychology, 1953, 46, 373-380.

Gravetter, F.. \& Lockhend, G. R. Criterial range as a frame of reference for stimulus judgment. Psychological Review, 1973, 80, 203-216.
Green, D. M., \& LUCE, R. D. Variability of magnitude estimates: A timing theory analysis. Perception \& Psychophysics, 1974, 15, 291-300.

LUCE, R. D., \& GREEN, D. M. A neural timing theory for response times and the psychophysics of intensity. Psychological Review, 1972, 79, 14-57.

LuCE, R. D., \& GREEN, D. M. The response ratio hypothesis for magnitude estimation. Journal of Mathematical Psychology, 1974, 11, 1-14. (a)

Luce, R. D., \& Green, D. M. Neural coding and psychophysical discrimination data. Journal of the Acoustical Society of America, 1974, 56, 1554-1564. (b)

Miller, G. A. The magical number seven, plus or minus two: Some limits on our capacity for processing information. Psychological Review, 1956, 63, 81-97.

Pollack, I. Information of elementary auditory displays. Journal of the Acoustical Society of America, 1952, 24. 745-750.

\section{NOTE}

1. We express our thanks to Stephen Burbeck and Brian Wandell, who wrote the computer programs and carried out all of the computations reported.

(Received for publication February 16. 1976; revision received April 20, 1976.) 